\title{
Application of the new Monte Carlo code AlfaMC to the calibration of alpha-particle sources
}

\author{
Miguel Jurado Vargas, \\ Alfonso Fernández Timón, \\ Carlos García Orellana
}

\begin{abstract}
Measurements of $\alpha$-particle sources require corrections to the counting rate due to scattering and self-absorption in the source and the backing material. In this study, we describe a simple procedure to estimate these corrections using the new Monte Carlo code AlfaMC to consider the effects of scattering and self-absorption conjointly, and so to determine the activity of $\alpha$ emitters. The procedure proposed was applied to ${ }^{235} \mathrm{UO}_{2}$ sources deposited on highly polished platinum backings. In general, the dependence of the efficiency with source thickness was in good agreement with a simple model considering a linear and a hyperbolic behavior for thin and thick sources, respectively, although significant deviations from this model were found for very thin sources. For these very thin sources, the Monte Carlo simulation revealed to be as a required method in the primary calibration of $\alpha$-particle sources. The efficiency results obtained by simulation with AlfaMC were in agreement with available efficiency data.
\end{abstract}

Key words: alpha-particle spectrometry $\bullet$ code AlfaMC $\bullet$ Monte Carlo simulation $\bullet$ scattering and self-absorption

M. Jurado Vargas ${ }^{\bowtie}$

Departamento de Física,

Universidad de Extremadura,

Avda. de Elvas s/n, 06006, Badajoz, Spain,

Tel.: +34 924289 537, Fax: +34 924289651 ,

E-mail:mjv@unex.es

A. Fernández Timón

Departamento de Informática y Estadística,

Universidad Rey Juan Carlos,

c/Tulipán s/n, 28933, Móstoles-Madrid, Spain

C. García Orellana

Departamento de Ingeniería Eléctrica, Electrónica y

Automática,

Universidad de Extremadura,

Avda. de Elvas s/n, 06006, Badajoz, Spain

Received: 24 September 2014

Accepted: 20 May 2015

\section{Introduction}

The efficiency (counting rate/activity) for $\alpha$-particle sources measured using detection systems with $2 \pi$ counting geometry can deviate significantly from $50 \%$, due to the backscattering in the source backing and to the scattering and self-absorption into the source substrate. In order to accurately calibrate $\alpha$-particle sources, it is necessary to make corrections to the counting rate. These corrections have been theoretically studied by several workers [1-5], generally assuming models that take into account the multiple scattering of $\alpha$ particles, most of them based on the general theory of multiple scattering from Williams [6]. These corrections can also be estimated experimentally [7-10], but it requires the measurement of a great number of sources prepared with a wide range of thicknesses and using a substrate with a similar chemical composition to the source of interest. An alternative method to calculate the desired corrections to the counting rate in $2 \pi$ detection systems is the use of Monte Carlo simulation to model the behavior of the $\alpha$ particles in the source and backing. Some works have been published using this procedure [11-14], using the well-known code SRIM [15].

In this study, we applied the recently developed code AlfaMC [16] to the determination of the corrections needed in the standardization of $\alpha$-particle sources measured with $2 \pi$ detection systems. Most 
of the Monte Carlo codes are generalist, so that the computational charge to simulate specifically the $\alpha$-particle transport is very high, resulting in very slow calculations. AlfaMC is specific for $\alpha$-particle transport, so that it uses more simple models, allowing also the implementation of complex geometries. As a consequence, all its calculations are more rapid with respect to more complex codes. The transport of the $\alpha$ particles is based on the continuous slowing down approximation (CSDA), with no secondary particles being produced. The average energy losses per unit length are calculated by taking into account the stopping powers supplied by the NIST ASTAR database [17], and the energy straggling is considered to be a simple Gaussian process, although a more detailed model, taking into consideration the Landau and Vavilov theory [18], can also be included in the simulations. The code adopts the theory of Fermi to describe the multiple scattering of $\alpha$ particles, which is a valid approximation in the energy range from 1 to $12 \mathrm{MeV}$.

\section{Simulation procedure}

A simulation of $\alpha$ particles in deposits of ${ }^{235} \mathrm{UO}_{2}$ mounted on a highly polished platinum backing was performed. The sources have an active area of $1.267 \mathrm{~cm}^{2}$ (12.7 $\mathrm{mm}$ diameter), and are assumed to be measured in a $2 \pi$ detection system (see Fig. 1). The characteristics of these sources correspond to the same considered in the experimental work of Hutchinson et al. [9] for comparison purposes. In order to simplify the simulations, homogeneous sources of pure ${ }^{235} \mathrm{U}$ were chosen, while the content of ${ }^{235} \mathrm{U}$ in the experimental sources was of $99.7 \%$.

Because ${ }^{235} \mathrm{U}$ has many $\alpha$ emissions, each $\alpha$-particle group was split into several sets with a number of simulated particles proportional to the corresponding emission probabilities. Only the ${ }^{235} \mathrm{U}$ alpha lines with emission probabilities greater than $0.5 \%$ were simulated, which were taken from the LNHB database [19]. The deposit and backing surfaces were considered to be flat, so that surface effects due to irregularities were not included in the simulation. The initial directions were randomly distributed over a solid angle of $4 \pi$ sr, so that the particle tracks were followed into both source and backing. Each $\alpha$ particle was followed until it was finally emitted from the source into the $2 \pi$ chamber or when its energy was totally lost in the $\mathrm{UO}_{2}$ substrate or platinum backing. The main characteristics

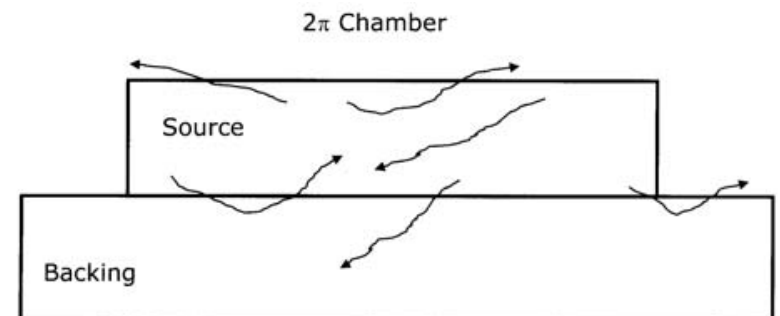

Fig. 1. Scheme of the simulation performed in this work for the ${ }^{235} \mathrm{UO}_{2} \alpha$-particle sources. of the particle at each step of the track, as energy, direction and position, are available, and the total track can be depictured.

\section{Results and discussion}

\section{Application to thin and thick sources}

Source thicknesses from 1 to $40 \mathrm{mg} / \mathrm{cm}^{2}$ were studied, in order to include thin and thick sources. Figure 2 shows the obtained $\alpha$-particle spectra for sources with some very different substrate thicknesses. It is evident the increasing overlap of the ${ }^{235} \mathrm{U} \alpha$ emissions and the greater tails in the low-energy zone when the source thickness is increased, as a consequence of the loss of energy of the $\alpha$ particles in the source deposit (self-absorption). In each spectrum, there is a 'maximum' at the low-energy region where the $\alpha$-particle energy is near to zero. This fact was already analyzed by us in a previous study [13] and is due to the backscattered particles that have undergone a very large number of weak collisions with the atomic electrons in the source substrate (multiple scattering).

For each simulation, the detection efficiency was determined by the ratio between the number of detected particles (leaving upwards the source into a $2 \pi$ sr solid angle) and the initial number of simulated particles. Changing the random number seeds, we obtained different results for estimating the uncertainty related to each efficiency value. Each source with a given thickness was simulated three times, each including $9 \times 10^{4} \alpha$ particles, with a mean calculation time of about $2 \mathrm{~min}$. The corresponding efficiency values, jointly with their uncertainties, are depicted in Fig. 3.

A simple theoretical model can be used to describe the dependence of the detection efficiency respect to the source thickness $[4,5]$, by assuming straight lines for the tracks of $\alpha$ particles into the substrate and backing, that is, when the scattering is not considered. Following this model, a linear dependence on source thickness is obtained for thin samples, while for thick samples, the dependence on thickness is described by a power law, which is simplified to a hyperbolic behavior if the source

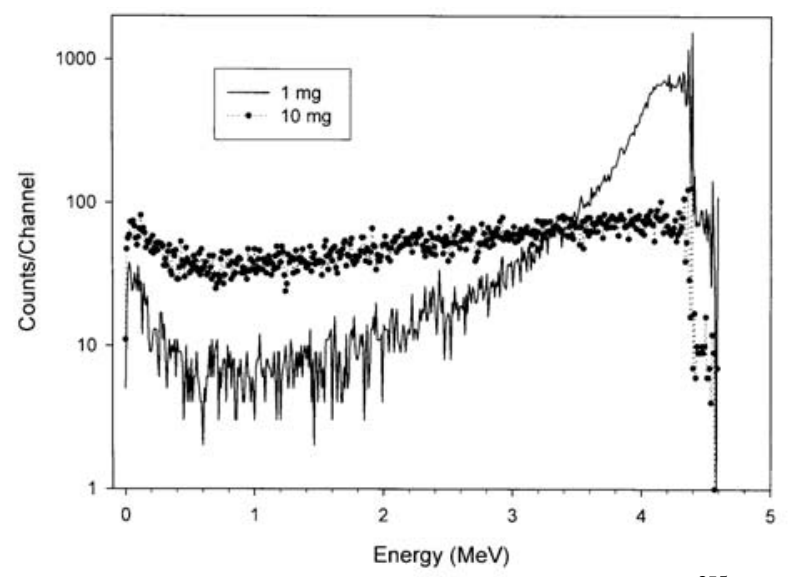

Fig. 2. $\alpha$-Particle spectra obtained for sources of ${ }^{235} \mathrm{UO}_{2}$ with different source thicknesses. Spectra are plotted with a $10 \mathrm{keV}$ energy interval. 


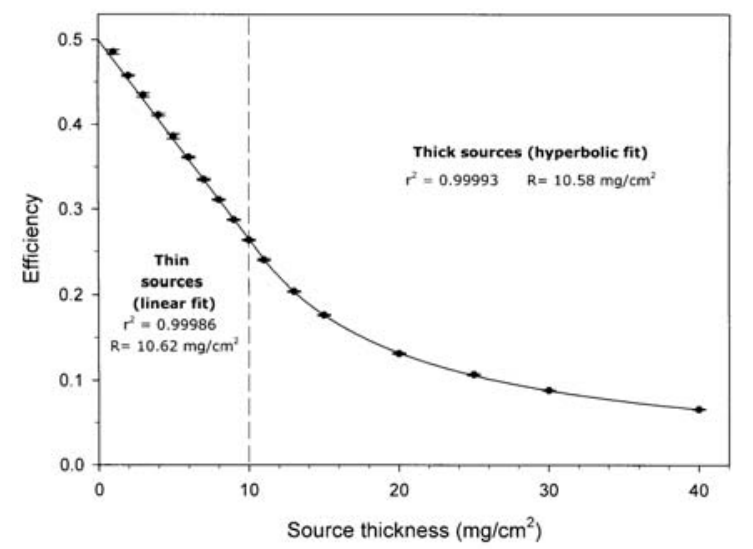

Fig. 3. Values of efficiency obtained for thin and thick $\alpha$-particle sources of ${ }^{235} \mathrm{UO}_{2}$ vs. the source thickness. The uncertainties shown correspond to one standard deviation. Lines represent the fittings of each curve to the expressions (1) and (2) for the regions of thin and thick sources, respectively.

surface is perfectly flat. Therefore, the dependence of $\alpha$ efficiency $\varepsilon$ on the source thickness $d$ can be described by:

$$
\begin{gathered}
\varepsilon(d)=\frac{1}{2}\left(1-\frac{d}{2 R}\right), \text { when } d \leq R \\
\varepsilon(d)=\frac{R}{4 d}, \text { when } d \geq R
\end{gathered}
$$

where $R$ is the mean range of $\alpha$ particles in the $\mathrm{UO}_{2}$ substrate, and $d$ denotes the substrate thickness.

Because the theoretical model assumes straight lines for $\alpha$ particles, the most appropriate mean range $R$ to be considered in the equations must be the CSDA range, which represents the average path length traveled by the $\alpha$ particle as it slows down to rest, by considering the CSDA. Data of the CSDA range for $\alpha$ particles in $\mathrm{UO}_{2}$ are not available, so that we carried out a calculation by integrating the reciprocal of the total stopping power for $\mathrm{UO}_{2}$ with respect to energy. The total stopping powers for $\mathrm{UO}_{2}$ in the energy range from 0 to $4.4 \mathrm{MeV}$ were derived from the corresponding values for $\mathrm{U}$ and $\mathrm{O}$ in the ASTAR database program [17] using the Bragg's additivity rule. The final CSDA range thus obtained for $4.4 \mathrm{MeV}$ $\alpha$ particles (mean energy for the ${ }^{235} \mathrm{U}$ emissions) in $\mathrm{UO}_{2}$ was $10.87 \mathrm{mg} / \mathrm{cm}^{2}$.

Expressions (1) and (2) were independently fitted to the zones of thin $\left(d<11 \mathrm{mg} / \mathrm{cm}^{2}\right)$ and thick thickness $\left(d \geq 11 \mathrm{mg} / \mathrm{cm}^{2}\right)$, respectively, by taking as a free parameter the mean range $R$. In order to fit the zone of thin thicknesses, it was imposed an efficiency value of 0.5 for a source with null thickness. The corresponding linear fittings are shown in Fig. 3 superimposed to the simulated efficiency values.

At first sight, we can see that the proposed linear function fit well the efficiency values for thin sources (up to $10 \mathrm{mg} / \mathrm{cm}^{2}$ ), giving a correlation coefficient very close to unity and a mean range $R$ of $10.62 \mathrm{mg} / \mathrm{cm}^{2}$, a value close to the CSDA range of $10.87 \mathrm{mg} / \mathrm{cm}^{2}$. Some small deviations from the theoretical linear behavior in the region of small thicknesses ( 1 up to $4 \mathrm{mg} / \mathrm{cm}^{2}$ ) are present, as shown in column 3 of Table 1, which can be explained in terms
Table 1. Values of the efficiency obtained for thin and thick sources of ${ }^{235} \mathrm{UO}_{2}$. The uncertainties correspond to one standard deviation. Thin and thick sources are separated with a dashed line. The relative deviations with respect to the fits to expressions (1) and (2) are also shown

\begin{tabular}{rcc}
\hline $\begin{array}{c}\text { Source thickness } \\
{\left[\mathrm{mg} / \mathrm{cm}^{2}\right]}\end{array}$ & Efficiency & $\begin{array}{c}\text { Deviation } \\
{[\%]}\end{array}$ \\
\hline 1 & $0.4855 \pm 0.0024$ & 1.89 \\
2 & $0.4574 \pm 0.0010$ & 0.97 \\
3 & $0.4344 \pm 0.0023$ & 1.14 \\
4 & $0.4110 \pm 0.0015$ & 1.23 \\
5 & $0.3858 \pm 0.0029$ & 0.86 \\
6 & $0.3613 \pm 0.0009$ & 0.64 \\
7 & $0.3351 \pm 0.0010$ & -0.12 \\
8 & $0.3111 \pm 0.0008$ & -0.29 \\
9 & $0.2874 \pm 0.0006$ & -0.38 \\
10 & $0.2634 \pm 0.0008$ & -0.60 \\
11 & $0.2399 \pm 0.0012$ & -0.24 \\
13 & $0.2038 \pm 0.0007$ & 0.16 \\
15 & $0.1763 \pm 0.0008$ & -0.03 \\
20 & $0.1314 \pm 0.0006$ & -0.65 \\
25 & $0.1069 \pm 0.0011$ & 1.03 \\
30 & $0.0883 \pm 0.0003$ & 0.14 \\
40 & $0.0661 \pm 0.0005$ & -0.05 \\
\hline
\end{tabular}

of the scattering effects of $\alpha$ particles in both the source substrate and the platinum backing, which are considered in the simulation code AlfaMC, but not in the simplified theoretical model.

The region of thick sources $\left(d \geq 11 \mathrm{mg} / \mathrm{cm}^{2}\right)$ was fitted by Eq. (2), obtaining an excellent fit, with a correlation coefficient very close to unity. As seen from Table 1, the relative deviations of the calculated efficiencies from the hyperbolic dependence were always below $1.1 \%$ (see Table 1 ). The derived value from this fit for the mean range $R$ was $10.58 \mathrm{mg} / \mathrm{cm}^{2}$, a value extremely close to that obtained in the fit to thin sources and also very similar to the CSDA range. The agreement between the simulation and the theoretical model for the region of thick sources is some better than that obtained for thin sources, because the scattering effects do not contribute greatly on the detection efficiency in sources with thicknesses greater than the mean range $R$.

All these considerations show that the dependence of the efficiency curve obtained with AlfaMC with respect to the source thickness at both ranges of thin and thick sources is approximately well followed by this simplified theoretical model. Finally, we have checked that for sources with thicknesses above $2 \mathrm{mg} / \mathrm{cm}^{2}$, all the backscattered particles in the platinum backing are finally absorbed into the substrate, and finally, do not go out towards the detection chamber. Then, the contribution of the backscattering in the platinum backing on the detection efficiency is null for these sources.

\section{Application to very thin sources}

In order to study the efficiency behavior for the cases of very thin deposits, ${ }^{235} \mathrm{UO}_{2}$ homogeneous sources with thicknesses in the range from 0 to 


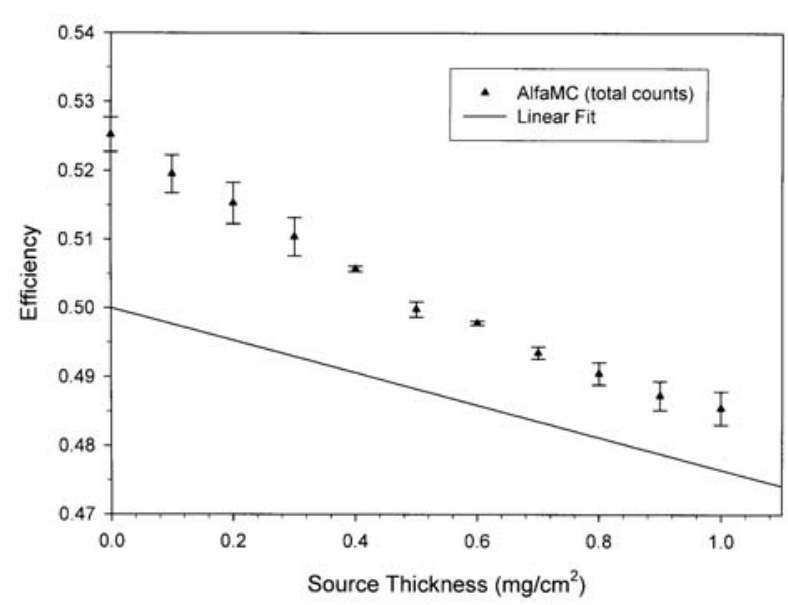

Fig. 4. Values of efficiency obtained for very thin $\alpha$-particle sources of ${ }^{235} \mathrm{UO}_{2}$ vs. the source thickness. The uncertainties shown correspond to one standard deviation. The straight line shown corresponds to the linear fit carried out before in Fig. 3.

$1 \mathrm{mg} / \mathrm{cm}^{2}$ were simulated. In addition, in this range, our simulated efficiencies can be compared with the available experimental values of efficiency obtained by Hutchinson et al. [9]. Figure 4 shows the efficiency results derived from these new simulations, together with the straight line from the fit carried out before in Fig. 3. As before, each value corresponds to the mean of three simulations, each including different random number seeds and $9 \times 10^{4}$ initial $\alpha$ particles. It is clearly evidenced that the linear behavior established in the theoretical model is totally inadequate for accounting the efficiency behavior in the region of smaller thicknesses. Therefore, for sources in this thickness range, the application of Monte Carlo procedures is specially required in the calibration of $\alpha$-particle sources to include properly the scattering effects in the substrate and backing of the source. It must be noted that, in this range of very thin sources, many of the backscattered particles in the platinum backing go out towards the detection chamber, so that the contribution of the backscattering in the backing on the detection efficiency is significant for sources with very thin thicknesses. This can be seen in Table 2, which

Table 2. Percentage of the detected $\alpha$ particles being backscattered in the platinum backing with respect to the total detected backscattered particles (backscattered in both substrate and backing)

\begin{tabular}{|c|c|}
\hline $\begin{array}{c}\text { Source thickness } \\
{\left[\mathrm{mg} / \mathrm{cm}^{2}\right]}\end{array}$ & $\begin{array}{c}\text { Percentage } \\
{[\%]}\end{array}$ \\
\hline 0 & 100.0 \\
\hline 1 & 71.0 \\
\hline 2 & 59.1 \\
\hline 3 & 47.4 \\
\hline 4 & 36.8 \\
\hline 5 & 27.4 \\
\hline 6 & 21.4 \\
\hline 7 & 12.8 \\
\hline 8 & 8.2 \\
\hline 9 & 5.1 \\
\hline 10 & 2.6 \\
\hline
\end{tabular}

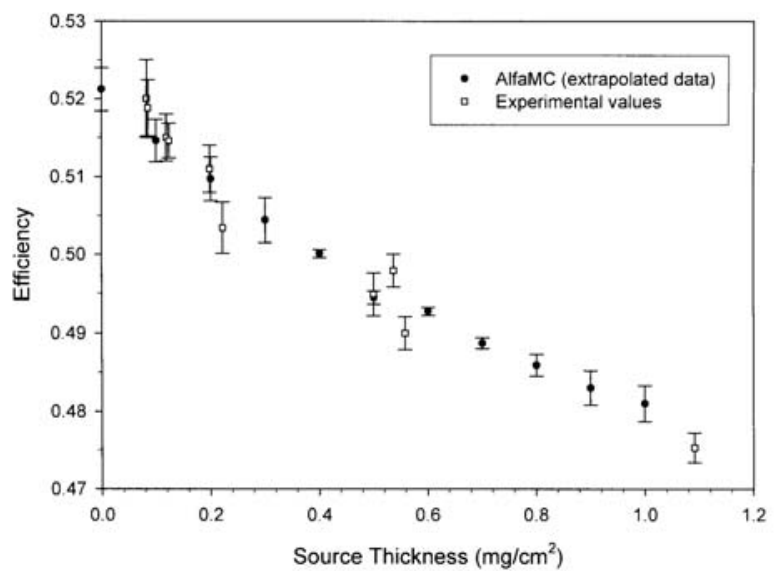

Fig. 5. Values of efficiency obtained for very thin $\alpha$-particle sources of ${ }^{235} \mathrm{UO}_{2}$ after performing a zero-energy extrapolation. Experimental values obtained by Hutchinson et al. [9] are also shown for comparison purposes. All the uncertainties shown correspond to one standard deviation.

shows the percentage of $\alpha$ particles backscattered in the platinum backing that are detected with respect to the total detected backscattered particles.

In order to compare our efficiency results with that obtained experimentally by Hutchinson et al. [9], a zero-energy extrapolation must be performed for each corresponding simulated spectrum. The reason for it is the fact that in experimental measurements, only the pulses above a given energy cut-off are considered because the $\alpha$ particles in the low-energy zone of the pulse-height distribution cannot be distinguished from pulses produced by recoiling daughters and by electronic noise. Then, in order to estimate the total $2 \pi$ counting rate up to zero energy, an extrapolation to zero energy is usually performed in the experimental spectrum. These 'extrapolated values' for the efficiency are shown in Fig. 5, together with the experimental efficiencies given in the work of Hutchinson et al. [9]. The 'extrapolated values' are somewhat smaller than the 'total values' for the efficiency, as a consequence of the 'maximum' found at the low-energy region of the simulated spectra (see Fig. 2). In addition, the simulated efficiencies (extrapolated to zero energy) seem to follow, in general, a similar trend to the experimental values. The agreement is satisfactory taking into consideration the experimental uncertainties.

\section{Conclusions}

In this work, a study has been carried out in order to check the applicability of the code AlfaMC to the tasks of primary calibration of $\alpha$-particle sources using $2 \pi$ detectors. The values of the detection efficiency (counting rate/activity) were derived by simulation for homogeneous sources of ${ }^{235} \mathrm{UO}_{2}$ over a wide range of thicknesses (from 0 to $40 \mathrm{mg} / \mathrm{cm}^{2}$ ) mounted on a platinum backing. In general, the dependence of the efficiency with source thickness was in good agreement with a simple model considering a linear and a hyperbolic behavior for thin and thick sources, respectively. However, significant deviations from this simplified model were found 
for very thin sources, where the scattering effects in the substrate and in the backing have an important contribution to the detection efficiency. For these very thin sources, the Monte Carlo simulation has revealed as a required method in the primary calibration of $\alpha$-particle sources. The efficiency results obtained by simulation with AlfaMC for the thickness range used in the work by Hutchinson et al. [9] were in agreement with the experimental efficiency data.

This work shows that the code AlfaMC can be used as a simple and rapid procedure to determine jointly the corrections for scattering and self-absorption required in the measurement of $\alpha$-particle sources.

Acknowledgments. Thanks are due to Luis Peralta and Alina Louro for providing us with the version of the code AlfaMC. Thanks are due to the Gobierno de Extremadura (projects GRU09011 and GR10160) and Ministerio de Economía y Competitividad (project TIN2012-35632-C02-02) for financial support. We would like to thank Mrs. María Victoria Jurado Vargas for the revision of the English text.

\section{References}

1. Crawford, J. A. (1949). Theoretical calculations concerning backscattering of alpha particles. In The transuranium elements (Part II, pp. 1307-1326). New York: McGraw-Hill.

2. Lucas, L. L., \& Hutchinson, J. M. R. (1976). Study of the scattering correction for thick uranium-oxide and other $\alpha$-particle sources - I: Theoretical. Appl. Radiat. Isot., 27, 35-42.

3. Rossi, B. B., \& Staub, H. H. (1949). Ionization chambers and counters. Experimental techniques. New York: McGraw-Hill.

4. Semkow, T. M., Jeter, H. W., Parsa, B., Parekh, P. P., Haines, D. K., \& Bari, A. (2005). Modeling of alpha mass-efficiency curve. Nucl. Instrum. Methods Phys. Res. Sect. A-Accel. Spectrom. Dect. Assoc. Equip., 538, 790-800.

5. White, P. H. (1970). Alpha and fission counting of thin foils of fissile material. Nucl. Instrum. Methods, $79,1-12$.
6. Williams, E. J. (1940). Multiple scattering of fast electrons and alpha particles, and "curvature" of cloud tracks due to scattering. Phys. Rev., 58, 292-306.

7. Ballaux, C. (1985). Note on the scattering corrections in $2 \pi \alpha$ counting. Appl. Radiat. Isot., 36, 822-824.

8. Deruytter, A. J. (1962). Evaluation of the absolute activity of alpha emitters and of the number of nuclei in thin alpha active layers. Nucl. Instrum. Methods, 15, 164-170.

9. Hutchinson, J. M. R., Lucas, L. L., \& Mullen, P. A. (1976). Study of the scattering correction for thick uranium-oxide and other $\alpha$-particle sources - II: Experimental. Appl. Radiat. Isot., 27, 43-45.

10. Walker, D. H. (1965). An experimental study of the backscattering of 5.3-MeV alpha particles from platinum and monel metal. Appl. Radiat. Isot., 16, 183-189.

11. Fernández Timón, A., Jurado Vargas, M., \& Ziegler, J. F. (2014). Application of alpha particle transport to the modelization of efficiency curves in proportional counters. J. Radioanal. Nucl. Chem., 302, 297-302.

12. Ferrero, J., Roldán, C., Aceña, M., \& García-Toraño, E. (1990). Backscattering and self-absorption corrections in the measurement of alpha-emitters in $2 \pi$ geometry. Nucl. Instrum. Methods Phys. Res. Sect. AAccel. Spectrom. Dect. Assoc. Equip., 286, 384-387.

13. Jurado Vargas, M., \& Fernández Timón, A. (2004). Scattering and self-absorption corrections in the measurement of $\alpha$-particle emitters in $2 \pi$ geometry. $\mathrm{Nucl}$. Instrum. Methods Phys. Res. Sect. B-Beam Interact. Mater. Atoms, 217, 564-571.

14. Jurado Vargas, M., \& Fernández Timón, A. (2005). Dependence of self-absorption on thickness for thin and thick alpha-particle sources of $\mathrm{UO}_{2}$. Nucl. Instrum. Methods Phys. Res. Sect. A-Accel. Spectrom. Dect. Assoc. Equip., 548, 432-438.

15. Ziegler, J. F., Biersack, J. P., \& Littmark, U. (1985). The stopping and range of ions in solids. New York: Pergamon Press.

16. Peralta, L., \& Louro, A. (2014). AlfaMC: A fast alpha particles transport Monte Carlo code. Nucl. Instrum. Methods Phys. Res. Sect. A-Accel. Spectrom. Dect. Assoc. Equip., 737, 163-169.

17. Berger, M. J., Coursey, J. S., Zucker, M. A., \& Chang, J. (2011). Stopping-power and range tables for helium ions. Retrieved April 24, 2014, from http://physics. nist.gov/PhysRefData/Star/Text/ASTAR.html.

18. Vavilov, P. V. (1957). Ionization losses of high-energy heavy particles. Sov. Phys. JETP, 5, 749-751.

19. Laboratoire National Henri Becquerel. (2008). Atomic \& Nuclear Data. Retrieved April 24, 2014, from http://www.nucleide.org/NucData.htm. 